\title{
DEVELOP A MODEL TO STUDY THE ENERGY DISTRIBUTION OF CASCADES OF ATOMIC COLLISIONS
}

\author{
A.A. Aleksandrov ${ }^{1}$ \\ rector@bmstu.ru \\ V.A. Akatev ${ }^{1}$ \\ akatiev07@mail.ru \\ E.V. Metelkin ${ }^{2}$ \\ sitech47@mail.ru \\ E.Yu. Baryscheva ${ }^{1}$ \\ barykaterina@yandex.ru \\ ${ }^{1}$ Bauman Moscow State Technical University, Moscow, Russian Federation \\ ${ }^{2}$ Russian State Social University, Moscow, Russian Federation
}

\begin{abstract}
In this paper, based on the solution of the Boltzmann kinetic equation, we determine the energy distribution function describing the steady-state deceleration of the cascade of moving atoms taking into account their multiplication at the power interaction potential $U \sim 1 / r^{n}$. A new approach to the solution of the kinetic equation based on the extended concept of primary knocked-on atoms (PKA) is used for its calculation. One of the advantages of using the power interaction potential is that in this case it is possible to obtain simple analytical formulas for the distribution function of the cascade of slowing-down atoms taking into account their multiplication and demonstrate the simplicity and convenience of the proposed new approach to the solution of the kinetic equation. On the other hand, based on the obtained results it is possible to estimate the accuracy of various approximate solutions. It is shown that this approach will be applicable to other interatomic interaction potentials, if the average PKA energy loss in individual collisions decreases monotonically with decreasing energy, and the relative PKA energy loss in individual collisions will be small
\end{abstract}

\section{Keywords}

Kinetic equation, collisions, cascade of atoms, slowing-down of atoms, interaction potential

Received 24.04.2018

(c) Author(s), 2019

The development of nuclear reactors and thermonuclear installations involves the selection of radiation-resistant materials as their bodies and individual elements must withstand long-term exposure to radiation. Irradiation of solids by fast particles (neutrons, fast ions) leads to the fact that the atoms of the crystal lattice received from the incoming particle energy greater than a certain threshold 
A.A. Aleksandrov, V.A. Akatev, E.V. Metelkin, E.Yu. Baryscheva

value fly out of their equilibrium positions. Further, the exchange of energy between moving atoms and atoms located in the nodes of the crystal lattice leads to the emergence of new generations of knocked-on atoms. Thus, there is a socalled cascade of atomic collisions. As a result of the cascade development the primary point defects (vacancies, interstitial atoms) are formed in the solid body, which determine the accumulation of radiation damage in irradiated materials and subsequent changes in their physical and mechanical properties $[1,2]$.

For the correct description of these processes, it is necessary the information on the nature of the energy distribution of the decelerating cascade of moving atoms. A significant number of works are devoted to the study of the development of atomic collision cascades in a solid, for example [3-9]. To be noticed is that the authors of works $[7,8]$ found a distribution function that describes the energy distribution of a cascade of moving atoms taking into account their multiplication for the simplest special case of elastic spherically symmetric scattering in the system of the center of mass without taking into account the binding energy of atoms in the lattice sites. There are no previous results for more complex interaction potentials of simple analytical solutions describing the cascade of decelerating atoms.

In this paper, based on the solution of the Boltzmann kinetic equation we determine the energy distribution function describing the steady-state deceleration of the cascade of moving atoms taking into account their multiplication at the power interaction potential $U \sim 1 / r^{n}$ [10]. A new approach to the solution of the kinetic equation based on the extended concept of primary knocked-on atoms (PKA) is used for its calculation. Henceforward, the PKA is that atom, which after the collision has more energy [6-9]. An atom with lower energy will be called the target atom (or knocked-on atom).

One of the advantages of using the power potential of interaction is the possibility of obtaining simple analytical formulas for the distribution function of the cascade of decelerating atoms taking into account their multiplication, which makes it possible to demonstrate the simplicity and convenience of the proposed below new approach to the solution of the kinetic equation. Using the results that we obtained it is also possible to estimate the accuracy of various approximate solutions.

Note that in the work [11] the authors determined the spatial and time characteristics of the primary knocked-on relativistic electrons (PKRE) decelerating in the substance due to ionization losses: the dependence of the PKRE energy on time, the dependence of the energy of the decelerating PKRE on the distance covered and the dependence of the distance covered by PKRE on the deceleration time. 
Problem statement. Consider a solid body consisting of atoms of the same type, whose binding energy at the nodes of the lattice is $\varepsilon_{d}$. Let us study in more detail the interaction between a moving atom and an atom located in a lattice node. Let the moving atom with energy $E^{\prime}$ as a result of collision with an atom of the crystal lattice becomes a state with energy $E$, by transmitting energy to the atom located in the node of the crystal lattice, $E^{\prime}-E-\varepsilon_{d}$. In this case, there is only one of two possible events can be realized: 1) the energy of a moving atom $E$ after elastic scattering, there may be more energy of the knocked-on atom $\left.E^{\prime}-E-\varepsilon_{d} ; 2\right)$ the energy knocked-on of an atom $E^{\prime}-E-\varepsilon_{d}$ there may be more energy from the moving atom after the impact.

The kinetic equation describing the unsteady energy distribution of moving atoms (both PKA and knocked-on) from an instantaneous uniformly distributed over the space of a monoenergy source without taking into account their collisions with each other is written as [12]

$$
\begin{gathered}
\frac{\partial f(E, t)}{\partial t}+\Sigma(E) \Phi(E, t)=\int_{E}^{E_{0}} d E^{\prime} \Sigma\left(E^{\prime} \rightarrow E\right) \Phi\left(E^{\prime}, t\right)+ \\
+\eta\left(E_{0}-E-\varepsilon_{d}\right) \int_{E+\varepsilon_{d}}^{E_{0}} d E^{\prime} \Sigma\left(E^{\prime} \rightarrow E^{\prime}-E-\varepsilon_{d}\right) \Phi\left(E^{\prime}, t\right)+N_{0} \delta\left(E \rightarrow E_{0}\right) \delta(t),
\end{gathered}
$$

where $f(E, t) d E$ is number of atoms with energy $E$ in the range $d E$ at time $t$ per unit volume; $\Sigma(E)$ is full macroscopic cross section of atomic scattering; $\Phi(E, t)=v f(E, t)$ is the flow of moving atoms, $v$ is the atoms' speed; $\Sigma\left(E^{\prime} \rightarrow E\right)$ is macroscopic cross section of atomic scattering; $\delta(x)$ is the Dirac Delta function; $N_{0}$ is total number of atoms; $E_{0}$ is initial energy of moving atoms; $\eta(x)$ is Heaviside unit function,

$$
\eta(x)=\left\{\begin{array}{l}
1, x>0 \\
0 ; x<0 .
\end{array}\right.
$$

The first integral on the right side of the kinetic equation (1) describes the transition of a moving atom with energy $E^{\prime}$ to the state with energy $E$. In this case, the knocked-on atom receives kinetic energy $E^{\prime}-E-\varepsilon_{d}$. The second integral describes the formation of a knocked-on atom with energy $E$, when a moving atom moved into a state with energy $E^{\prime}-E-\varepsilon_{d}$.

According to the terminology adopted above, we consider PKA to be the one that has more energy after each act of interaction. Obviously, contributions to the kinetic Eq. (1) from both the first and the second integral should be taken into account when the condition is met $E<E^{\prime}-E-\varepsilon_{d}$ or 
A.A. Aleksandrov, V.A. Akatev, E.V. Metelkin, E.Yu. Baryscheva

$E^{\prime}<2 E+\varepsilon_{d}$. In this case, the kinetic equation describing the energy distribution of PKA will be written as $[7,8]$ :

$$
\begin{gathered}
- \text { at } E \geq \frac{E_{0}-\varepsilon_{d}}{2} \\
\frac{\partial f(E, t)}{\partial t}+\Sigma(E) \Phi(E, t)=\int_{E}^{E_{0}} d E^{\prime} \Sigma\left(E^{\prime} \rightarrow E\right) \Phi\left(E^{\prime}, t\right)+ \\
+\eta\left(E_{0}-E-\varepsilon_{d}\right) \int_{E+\varepsilon_{d}}^{E_{0}} d E^{\prime} \Sigma\left(E^{\prime} \rightarrow E^{\prime}-E-\varepsilon_{d}\right) \Phi\left(E^{\prime}, t\right)+N_{0} \delta(t) \delta\left(E-E_{0}\right) ; \\
\text { - at } E \leq \frac{E_{0}-\varepsilon_{d}}{2} \\
\frac{\partial f(E, t)}{\partial t}+\sum(E) \Phi(E, t) \Sigma=\int_{E}^{2 E+\varepsilon_{d}} d E^{\prime} \Sigma\left(E^{\prime} \rightarrow E\right) \Phi\left(E^{\prime}, t\right)+ \\
+\int_{E+\varepsilon_{d}}^{2 E+\varepsilon_{d}} d E^{\prime} \Sigma\left(E^{\prime} \rightarrow E^{\prime}-E-\varepsilon_{d}\right) \Phi\left(E^{\prime}, t\right) .
\end{gathered}
$$

Based on the law of conservation of energy from Eq. (2) in the works [7, 8] the authors found that the average energy loss of PKA in a separate collision at $E^{\prime}>\varepsilon_{d}$ described by the expression for

$$
\Delta_{0}\left(E^{\prime}\right)=\int_{\left(E^{\prime}-\varepsilon_{d}\right) / 2}^{E^{\prime}} d E\left(E^{\prime}-E\right) P\left(E^{\prime} \rightarrow E\right)+\int_{0}^{\left(E^{\prime}-\varepsilon_{d}\right) / 2} d E\left(\varepsilon_{d}+E\right) P\left(E^{\prime} \rightarrow E\right),
$$

where $P\left(E^{\prime} \rightarrow E\right)=\Sigma\left(E^{\prime} \rightarrow E\right) / \Sigma\left(E^{\prime}\right)$ is the probability of transition of a moving atom in interaction with a stationary atom from a state with energy $E^{\prime}$ in a state with energy $E$ in a single interval of energies.

The result (3) has the following interpretation. Broken atoms can be formed when $E^{\prime}>\varepsilon_{d}$. If atom with energy $E^{\prime}$, colliding with the lattice atom acquires energy $E$ lying in the interval from $\left(E^{\prime}-\varepsilon_{d}\right) / 2$ till $E^{\prime}$, then it knocked-on the atom has less energy, and then it is not considered. In this case, the energy loss of the moving atom will be $\left(E^{\prime}-E\right)$, which reflects the first term in (3). If the incoming atom with energy $E^{\prime}$ as a result of the interaction, it moved into a state with energy $E$, lying in the interval $0 \ldots\left(E^{\prime}-\varepsilon_{d}\right) / 2$, the great energy acquired knocked-on atom, which are followed in the future. The atom itself with energy $E$ excluded from consideration, so that the loss of energy is $\left(E+\varepsilon_{d}\right)$, and what reflects the second term in the formula (3).

Exact analytical solution of Eq. (2) for a simple special case of elastic, spherically symmetric in the system of the center of mass of scattering of parti- 
cles with the same mass at $\varepsilon_{d}=0$ obtained in the works $[7,8]$. The solution of Eq. (2) for the power potential of interaction between atoms will be obtained below and the distribution function describing the steady-state deceleration of the cascade of moving atoms taking into account their multiplication will be determined on its basis.

Characteristics of the deceleration process of moving atoms. For the power potential of interaction between atoms $U \sim 1 / r^{n}$ the differential cross section of the scattering of a moving atom on the lattice atom is written as [10]

$$
\Sigma\left(E^{\prime} \rightarrow E\right)=\Sigma_{0} \frac{1}{E^{\prime m}\left(E^{\prime}-E\right)^{1+m}},
$$

where $\Sigma_{0}=$ const; $m=1 / n$.

Using (4), we find the complete macroscopic cross section

$$
\Sigma\left(E^{\prime}\right)=\int_{0}^{E^{\prime}-\varepsilon_{d}} \Sigma\left(E^{\prime} \rightarrow E\right) d E=\frac{\Sigma_{0}}{m E^{\prime m} \varepsilon_{d}^{m}}\left[1-\left(\frac{\varepsilon_{d}}{E^{\prime}}\right)^{m}\right] .
$$

In obtaining the formula (4) it was assumed that the energy of a moving atom exceeds the binding energy of the atom at the lattice site $E^{\prime}>\varepsilon_{d}$.

Using (4), (5), the formula (3) can determine the average energy loss of PKA energy in a separate collision. In a dimensionless form with $\Delta(\varepsilon)=\Delta_{0} / \varepsilon_{d}$ is

- at $m=1$

$$
\Delta(\varepsilon)=\frac{1}{1-\frac{1}{\varepsilon}}\left[\ln \left(\frac{\varepsilon}{4}\right)+2 \ln \left(1+\frac{1}{\varepsilon}\right)+1-\frac{1}{\varepsilon}\right]
$$

- at $m<1$

$$
\Delta(\varepsilon)=\frac{m \varepsilon^{1-m}}{\left[1-\left(\frac{1}{\varepsilon}\right)^{m}\right]}\left\{\frac{1}{m(1-m)}\left[2^{m}\left(1+\frac{1}{\varepsilon}\right)^{1-m}-1\right]-\frac{1}{m \varepsilon}\right\} .
$$

Here $\varepsilon=E^{\prime} / \varepsilon_{d}-$ the dimensionless energy.

The relative energy losses of the moving PKA in a separate collision are determined by the ratio

$$
\delta(\varepsilon)=\frac{\Delta(\varepsilon)}{\varepsilon} .
$$

Energy dependence of the average energy losses of PKA in individual collisions, constructed by formulas (6), (7) for various exponents $n$ (respectively 
$m=1 ; 2 / 3 ; 1 / 2 ; 2 / 5 ; 1 / 3 ; 1 / 4)$, shown in Figure, $a$. The average energy losses when decelerating PKA decreases and have a maximum value when the initial energy. This value can be found by formulas (6), (7), taking $\varepsilon \gg 1$ :

- at $m=1$

$$
\Delta_{m}=\ln \frac{\varepsilon_{0}}{4}+1
$$

- at $m<1$

$$
\Delta_{m}=\frac{\varepsilon_{0}^{1-m}\left(2^{m}-1\right)}{1-m} .
$$

It should be noted that the calculations carried out by the formulas (9), (10) are in good agreement with the results shown in the Figure, $a$.

According to the accepted terminology, PKA transmit energy to knockedon atoms, the latter appear only at energies $\varepsilon<\Delta_{m}$. Consequently, in the field of energy $\varepsilon>\Delta_{m}$ there are only PKA and in this area their distribution function describes the entire cascade of decelerating atoms. Thus, in the field of energy

$$
\Delta_{m} \leq \varepsilon \leq \varepsilon_{0}
$$

the energy distribution of the cascade of decelerating atoms taking into account their multiplication is described by the PKA distribution function, which is the solution of Eq. (2).

It should be noted that in the works $[7,8]$ the authors found exact solutions of Eq. (1), (2) for a simple special case of elastic, spherically symmetric scattering in the system of the center of mass without taking into account the binding energy of atoms in the lattice nodes. In this case, the PKA distribution function (solution of Eq. (2)) coincided in the energy region (11) with the energy distribution of the cascade of decelerating atoms taking into account their multiplication (solution of Eq. (1)).

The energy dependence of the relative energy losses of PKA in individual collisions, built on the formula (8) using the results (6), (7), is shown in the Figure, $b$. The relative energy losses of the decelerating PKA in individual collisions are small, which makes it possible to use the approximation of continuous energy losses to determine the distribution function of the decelerating PKA [12].

The stationary distribution function of decelerating the primary knocked-on atoms. Since the relative energy losses of the decelerating PKA in 

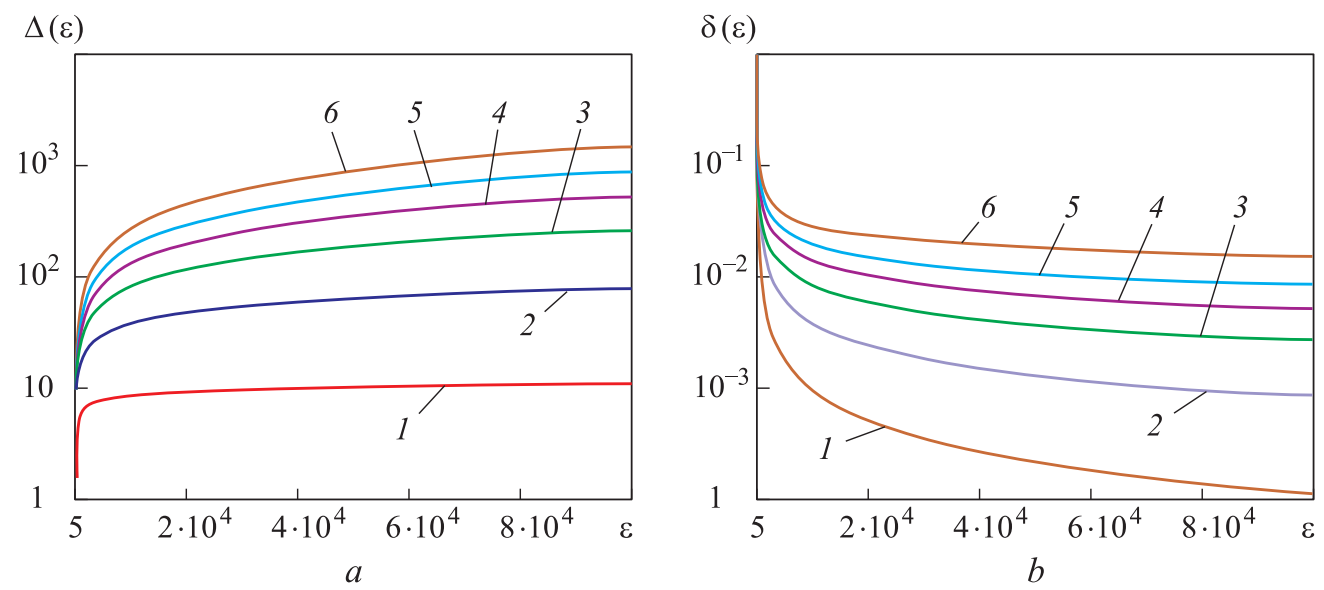

Energy dependences of average energy losses of PKA in separate collisions, constructed by formulas (6), (7) (a) and (8) using the results (6), (7) (b), class of PKA distribution functions $(c)$ at value $n=1(1), 1,5(2), 2(3), 2,5(4)$, $3(5), 4(6)$

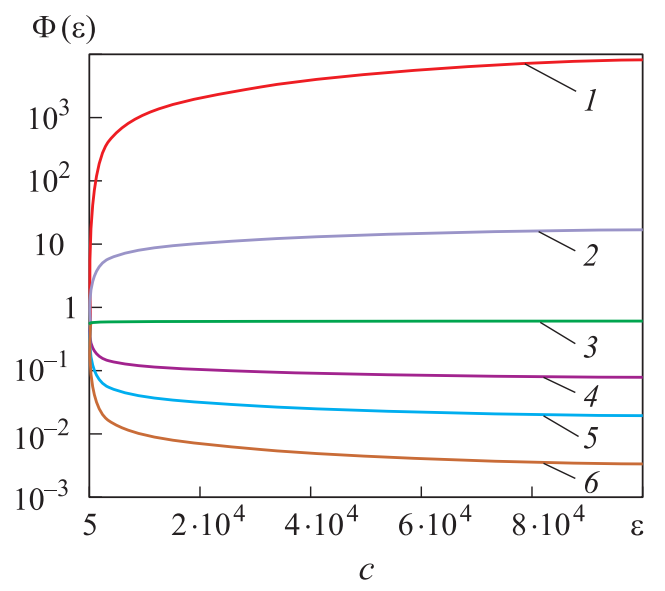

a separate collision are small, the approximation of continuous energy losses can be used to determine the stationary solution of Eq. (2) [12]. In this approximation, the stationary distribution function of decelerating PKA is written as [12]

$$
\Phi(E)=\frac{S_{0} \delta\left(E-E_{0}\right)}{\Sigma(E)}+\frac{S_{0}}{\Delta_{0}(E) \Sigma(E)},
$$

where $S_{0}$ is the number of PKA emitted by a source per unit time per unit volume.

In expression for (12), the first term describes the scattered radiation emitted by the source, i.e., atoms with energy $E_{0}$, and the second-the scattered radiation, i.e., atoms slowed down further to lower energies.

Using (5)-(7), the distribution function (12) can be represented in dimensionless form: 
- at $m=1$

$$
\begin{aligned}
& \Phi^{\prime}(\varepsilon)=\frac{m \varepsilon^{m}}{\left[1-\left(\frac{1}{\varepsilon}\right)^{m}\right]} \delta\left(\varepsilon-\varepsilon_{0}\right)+\frac{\varepsilon}{\left[\ln \left(\frac{\varepsilon}{4}\right)+2 \ln \left(1+\frac{1}{\varepsilon}\right)+1-\frac{1}{\varepsilon}\right]} ; \\
& - \text { at } m<1 \\
& \Phi^{\prime}(\varepsilon)=\frac{m \varepsilon^{m}}{\left[1-\left(\frac{1}{\varepsilon}\right)^{m}\right]} \delta\left(\varepsilon-\varepsilon_{0}\right)+\frac{\varepsilon^{2 m-1}}{\left\{\frac{1}{m(1-m)}\left[2 m\left(1+\frac{1}{\varepsilon}\right)^{1-m}-1\right]-\frac{1}{m \varepsilon}\right\}},
\end{aligned}
$$

where $\Phi^{\prime}=\Phi \Sigma_{0} /\left(S_{0} \varepsilon_{d}^{2 m-1}\right)$.

Family of PKA distribution functions (second term in (13), (14)) for several exponents $n$ shown in Figure, $c$. In the energy range (11), this result is the energy distribution of the cascade of decelerating atoms taking into account their multiplication.

Summary. In this paper, based on the solution of the Boltzmann kinetic equation we determine the energy distribution function describing the steadystate deceleration of the cascade of moving atoms taking into account their multiplication at the power interaction potential $U \sim 1 / r^{n}$ [10]. A new approach to the solution of the kinetic equation based on the extended concept of primary knocked-on atoms (PKA) is used for its calculation. This approach can also be applied to other interatomic interaction potentials if the average PKA energy losses in individual collisions decrease monotonically with decreasing energy, and the relative PKA energy losses in individual collisions are small. In this case, the distribution function describing the steady-state deceleration of the cascades of moving atoms taking into account their multiplication in the energy region (11) will be determined by the expression for (12), in which the values of $\Sigma(E)$ and $\Delta_{0}(E)$ are found by expressions for (3), (5).

Translated by K. Ivanova

\section{REFERENCES}

[1] Lehmann Chr. Interaction of radiation with solids and elementary defect production. Kernforschungsanlage Juelich, 1977.

[2] Was Gary S. Fundamentals of radiation materials science. Metals and alloys. Springer, 2010. 
[3] Ryazanov A.I., Metelkin E.V. Concerning the theory of radiation cascades of atomic collisions in a solid with an arbitrary interatomic interaction potential. Radiat. Eff., 1980, vol. 52, iss. 1-2, pp. 15-23. DOI: 10.1080/00337578008210012

[4] Satoh Y., Kojima S., Yoshiie T., et al. Criterion of subcascade formation in metals from atomic collision calculation. J. Nucl. Mater., 1991, vol. 179-181, part 2, pp. 901-904. DOI: 10.1016/0022-3115(91)90234-X

[5] Satoh Y., Yoshiie T., Kiritani M. Binary collision calculation of subcascade structure and its correspondence to observe subcascade defects in $14 \mathrm{MeV}$ neutron irradiated copper. J. Nucl. Mater., 1992, vol. 191-194, part B, pp. 1101-1105.

DOI: 10.1016/0022-3115(92)90645-2

[6] Metelkin E.V., Ryazanov A.I. Threshold energy of formation of subcascades. At. Energy, 1997, vol. 83, iss. 3, pp. 653-657. DOI: 10.1007/BF02415246

[7] Metelkin E.V., Ryazanov A.I., Semenov E.V. Developing new theoretical models of the formation of atomic collision cascades and subcascades in irradiated solids. J. Exp. Theor. Phys., 2008, vol. 107, no. 3, pp. 394-404. DOI: 10.1134/S1063776108090070

[8] Metelkin E.V., Ryazanov A.I., Semenov E.V. Ispol'zovanie kineticheskogo uravneniya Bol'tsmana dlya opisaniya ansamblya dvizhushchikhsya atomov, vybitykh iz uzlov kristallicheskoy reshetki, dlya issledovaniya obrazovaniya kaskadov i subkaskadov atomnykh stolknoveniy v obluchaemykh tverdykh telakh [Using Boltzmann kinetic equation for description of moving atoms assembly, knocked-out from lattice sites for study on formation of atomic collision cascades and subcascades in radiated solid bodies]. Preprint Kurchstov Institute. Moscow, NRC Kurchatov Institute Publ., 2008 (in Russ.).

[9] Ryazanov A.I., Metelkin E.V., Semenov E.V. Modeling of cascade and subcascade formation at high PKA energies in irradiated fusion structural materials. J. Nucl. Mater., 2009, vol. 386-388, pp. 132-134. DOI: 10.1016/j.jnucmat.2008.12.071

[10] Lindhard J., Vibeke Nielsen, Scharff M. Approximation method in classical scattering by screened Coulomb fields. Kopenhagen, Munksgaard, 1968.

[11] Akat'yev V.A., Metelkin E.V., Savinov A.M. Space-time characteristics of relativistic electrons moderated in matter. At. Energy, 2017, vol. 122, iss. 5, pp. 364-368.

DOI: $10.1007 / \mathrm{s} 10512-017-0280-6$

[12] Isakov A.I., Kazarnovskiy M.V., Medvedev Yu.A., et al. Nestatsionarnoe zamedlenie neytronov. Osnovnye zakonomernosti i nekotorye prilozheniya [Non-stationary moderation of neutrons. Main laws and some applications]. Moscow, Nauka Publ., 1984.

Aleksandrov A.A. - Dr. Sc. (Eng.), Professor, Rector of Bauman Moscow State Technical University, Head of Department of Ecology and Industrial Safety, Bauman Moscow State Technical University (2-ya Baumanskaya ul. 5, str. 1, Moscow, 105005 Russian Federation). 
A.A. Aleksandrov, V.A. Akatev, E.V. Metelkin, E.Yu. Baryscheva

Akatev V.A. - Dr. Sc. (Eng.), Professor, Department of Ecology and Industrial Safety, Bauman Moscow State Technical University (2-ya Baumanskaya ul. 5, str. 1, Moscow, 105005 Russian Federation).

Metelkin E.V. - Dr. Sc. (Phys.-Math.), Professor, Department of Technosphere Safety and Ecology, Russian State Social University (Vil'gel'ma Pika ul. 1, str. 4, Moscow, 129226 Russian Federation).

Baryscheva E.Yu. - Graduate Student, Department of Ecology and Industrial Safety, Bauman Moscow State Technical University (2-ya Baumanskaya ul. 5, str. 1, Moscow, 105005 Russian Federation).

\section{Please cite this article as:}

Aleksandrov A.A., Akatev V.A., Metelkin E.V., et al. Develop a Model to Study the Energy Distribution of Cascades of Atomic Collisions. Herald of the Bauman Moscow State Technical University, Series Natural Sciences, 2019, no. 1, pp. 27-36.

DOI: 10.18698/1812-3368-2019-1-27-36

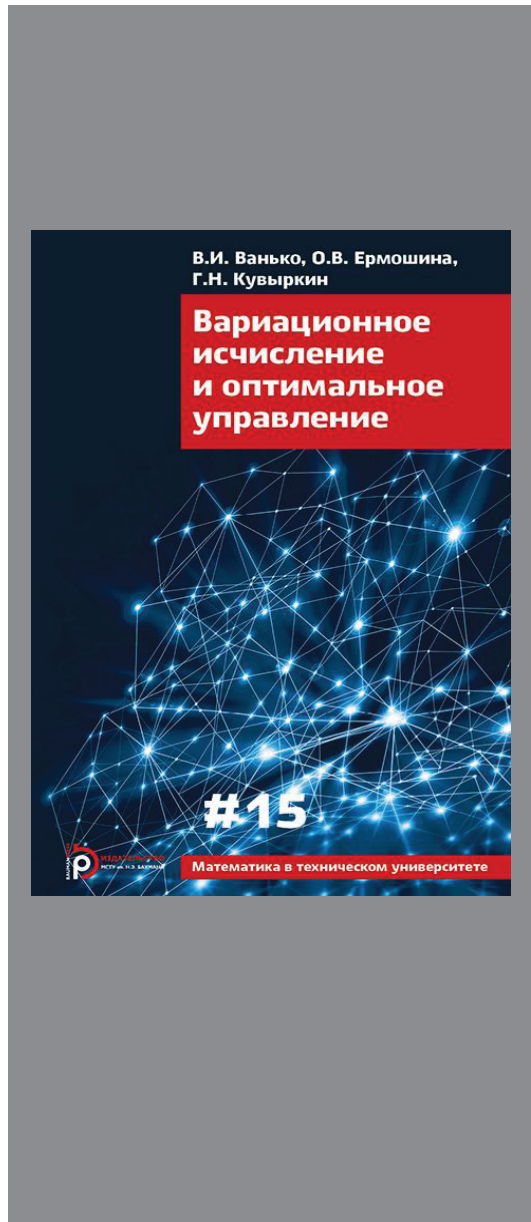

В Издательстве МГТУ им. Н.Э. Баумана вышел в свет учебник авторов

В.И. Ванько, О.В. Ермошиной, Г.Н. Кувыркина

\section{«Вариационное исчисление и оптимальное управление»}

Наряду с изложением основ классического вариационного исчисления и элементов теории оптимального управления рассмотрены прямые методы вариационного исчисления и методы преобразования вариационных задач, приводящие, в частности, к двойственным вариационным принципам. На примерах из физики, механики и техники показана эффективность методов вариационного исчисления и оптимального управления для решения прикладных задач.

Для студентов и аспирантов технических университетов, а также для инженеров и научных работников, специализирующихся в области прикладной математики и математического моделирования.

По вопросам приобретения обращайтесь: 105005, Москва, 2-я Бауманская ул., д. 5, стр. 1 +7 (499) 263-60-45

press@bmstu.ru

http://baumanpress.ru 